\title{
High-resolution spectroscopic imaging (HSI) mission
}

Fiona A. Harrison, Steven E. Boggs, Finn Erland Christensen, Neil A. Gehrels, Jonathan E. Grindlay, et al.

Fiona A. Harrison, Steven E. Boggs, Finn Erland Christensen, Neil A. Gehrels, Jonathan E. Grindlay, C. M. H. Chen, William W. Craig, Charles J. Hailey, Philip Pinto, Steven Thorsett, Jack Tueller, David L. Windt, Stanford E. Woosley, "High-resolution spectroscopic imaging (HSI) mission," Proc. SPIE 4851, X-Ray and Gamma-Ray Telescopes and Instruments for Astronomy, (11 March 2003); doi: 10.1117/12.461280

Event: Astronomical Telescopes and Instrumentation, 2002, Waikoloa, Hawai'i, United States 


\title{
The High-Resolution Spectroscopic Imaging (HSI) Mission
}

\author{
F. A. Harrison ${ }^{a}$, S. E. Boggs ${ }^{b}$, F. E. Christensen ${ }^{c}$, N. Gehrels ${ }^{d}$, J. E. Grindlay ${ }^{e}$ \\ C. M. H. Chen ${ }^{a}$, W. W. Craig $f$, C. J. Hailey ${ }^{g}$, P. Pinto ${ }^{h}$ \\ S. E. Thorsett ${ }^{i}$, J. Tueller ${ }^{d}$, D. Windt ${ }^{g}$, S. E. Woosley ${ }^{i}$ \\ ${ }^{a}$ Caltech Space Radiation Laboratory, 220-47 Pasadena, CA 91125 \\ ${ }^{b}$ Space Sciences Laboratory, U. C. Berkeley, Berkeley, CA \\ ${ }^{c}$ Danish Space Research Institute, Juliane Maries Vej 302100 Copenhagen, Denmark \\ ${ }^{d}$ Goddard Space Flight Center, Greenbelt, MD ${ }^{e}$ Harvard CfA, 60 Garden St., Cambridge, MA 02138 \\ $f$ Lawrence Livermore National Laboratory, Livermore, CA \\ ${ }^{g}$ Columbia Astrophysics Lab, Columbia University, Irvington, NY 10533, USA \\ ${ }^{h}$ Steward Observatory, University of Arizona, Tucson, AZ \\ ${ }^{i}$ Department of Astronomy and Astrophysics, University of California, Santa Cruz, CA 95064
}

\begin{abstract}
The High-Resolution Spectroscopic Imaging Mission is designed to be the first instrument to make true images of the hard X-ray/soft gamma-ray $(2-600 \mathrm{keV})$ sky. By focusing energetic X-rays and low-energy gamma-rays, HSI will observe the cosmos with an unprecedented combination of sensitivity, spectral resolution, and angular resolving power. HSI is based on an array of multilayer grazing-incidence optics focusing onto high-resolution solid-state germanium pixel detectors with a focal length of $30-50 \mathrm{~m}$. This paper describes the primary scientific objectives, technical approach to the instrumentation, and mission design.
\end{abstract}

\section{INTRODUCTION}

The High-Resolution Spectroscopic Imaging (HSI) Mission will carry the first instrument to make true images of the sky in the hard X-ray/soft gamma-ray band $(2-600 \mathrm{keV})$. HSI's ability to focus energetic X-rays and low-energy gamma-rays will enable it to observe the high-energy cosmos with an unprecedented combination of sensitivity, spectral resolution, and angular resolving power.

HSI has a number of primary scientific objectives: HSIs capabilities will help us to determine how Type 1a supernovae explode, providing the potential to understand the physical underpinnings of the observed width-luminosity relation required to employ SN1a's as probes of the geometry of the universe. Using sensitive hard x-ray observations with the arcsecond accuracy, HSI will trace the growth of galaxies and their nuclei over cosmic time. By studying the spatial and velocity distribution of recently-synthesized material in young supernova remnants, HSI will provide crucial information on how stars explode and distribute mass, heavy elements, and energy into galaxies. By observing magnetized neutron stars, relativistic shocks in gamma-ray burst sources, and high-energy particles in galaxy clusters, $H S I$ will study the sites of particle acceleration, and test physical theories under conditions unattainable in terrestrial laboratories. Finally, dramatic increases in flux sensitivity have always yielded major progress in astrophysics, and many of $H S T$ s contributions may well come from new discoveries.

HSI is an intermediate-scale mission, which will be primarily devoted to following up transients, and hard sources discovered by wide FOV survey telescopes. HST's $10-100$ fold improvements in sensitivity and angular and spectral resolution compared to other hard x-ray experiments result directly from focusing, which greatly reduces background compared to collimated or coded-aperture experiments, and allows the mission to employ compact, high-performance detectors. HSI builds on the technologies developed for the Constellation- $X$ mission, and the HEFT and InFoc $\mu \mathrm{s}$ balloon experiments. HSI is an natural follow-on to the Swift mission, and an essential complement to the EXIST survey ${ }^{1}$ which will provide arcminute locations for tens of thousands of threshold sources. Many hard X- Many hard

Further author information: (Send correspondence to Fiona A. Harrison)

E-mail: fiona@srl.caltech.edu 
X-ray sources are faint at other wavelengths, and without the arcsecond positions provided by HSI, their distances, and properties will remain unknown. HSI will also support the observations by $S N A P$ and $L S S T,{ }^{2}$ by studying the SN1a mechanism, and by following up interesting transients discovered by these wide-field optical imagers.

\section{SCIENTIFIC OBJECTIVES}

At energies below $10 \mathrm{keV}$, the emission from most astrophysical sources is dominated by thermal radiation from million-degree plasma. In contrast, at the hard X-ray/soft gamma-ray ( $5-600 \mathrm{keV})$ energies observed by HSI, radioactive decays from recently-synthesized material are observable, non-thermal processes often occurring in strong magnetic fields dominate, and emission can penetrate the dense material surrounding many black holes. Sources are, however, usually faint at these energies, and instrumental backgrounds high. The recent advent of hard X-ray optics that focus will enable observations 50 - 100 times more sensitive, with an order of magnitude better angular resolution and a factor 5 better spectral resolution than any other planned mission spanning this energy range.

Some of the HSI science goals are similar to the EXIST survey mission, and it is important to understand that the two are complementary: without the extensive catalog created by EXIST's all-sky survey, many fewer sources will be accessible for detailed study, and without HSI, many of the cataloged sources will remain unidentified, and their distance, luminosity and nature unknown.

\subsection{Type 1a Supernovae: Understanding the Explosion}

Over the past two decades a general consensus has arisen that Type 1a SNe result from the nuclear ignition of a white dwarf under extremely degenerate conditions, with a substantial fraction of its mass burned into nuclear statistical equilibrium in the process. ${ }^{3}$ In spite of significant theoretical progress in modeling the explosion, many fundamental parameters are unknown, and are unconstrained by observation. These include the composition and mass of the progenitor, the nature of the triggering process, the location of the initial detonation in the star, the propagation mechanism of the nuclear burning in the explosion, the resulting nuclear abundances, as well as the dynamics and structure of the ejecta, including the effects of instabilities in producing mixing or inhomogeneities.

The dominant nuclear product in Type $1 \mathrm{a}$ SNe is ${ }^{56} \mathrm{Ni}$, which decays with the emission of a $158 \mathrm{keV}$ gamma-ray. Measuring the ${ }^{56} \mathrm{Ni}$ abundance, and time evolution of the line width and flux is critical for understanding the nuclear burning processes. In addition, this decay provides a sensitive tool for understanding the dynamics of the explosion. Due to the similarity in ${ }^{56} \mathrm{Ni}$ decay time (8.8 days) and the typical timescale for the ejecta to become optically thin to the decay line emission ( 10 - 30 days), the $158 \mathrm{keV}$ emission line spans the transition of the ejecta from optically thin to optically thick, making them especially sensitive to the ejecta structure and dynamics, as well as the nuclear abundances and explosion mechanism of the SNe.,

HSI has the sensitivity, bandpass and spectral resolution to detect the ${ }^{56} \mathrm{Ni}$ line in Type 1a events out to the Virgo cluster, tracking the decay for months after the event. A few such observations over the course of the mission life would provide invaluable input to the theoretical explosions models, and a test of whether the observed luminosityduration correlation results from the mass of ${ }^{56} \mathrm{Ni}$, or from some other factor. This correlation is essential to the application of 1as to mapping dark energy, and $H S I$ has the potential to tell us if this relation is likely to evolve over cosmic time.

\subsection{Nucleosynthesis and Dynamics in Core Collapse Supernovae}

Core-collapse supernovae are likely the sites where the heavy elements are produced, and gamma-ray spectroscopy provides one of the few windows we have to study these explosions, and therefore the process of nucleosynthesis. Ideally one would like to observe core-collapse events in the months and years after the explosion, and observe the evolution of nuclear decays in the lightcurve. However these are significantly less luminous than Type 1as, and so currently-conceivable missions will be limited to rare (1/10 years) events within the local group. Luckily, nature has provided a radioactive tracer, observable in young remnants, that can provide many important diagnostics on the core-collapse explosion mechanism, nucleosynthesis, and dynamics. ${ }^{44} \mathrm{Ti}$ (decay lines at $68 \mathrm{keV}, 78 \mathrm{keV}$, and the $1157 \mathrm{keV}$ ) is produced in SNe near the "mass cut", or the spatial dividing line between the material that falls back onto the compact object, and the material that is ejected into the remnant. Its distribution in a young $(<2000$ year old) remnant, both in intensity and velocity as measured through doppler shifts of the lines, is very sensitive to the structure, dynamics, and mixing instabilities in the initial explosion. 


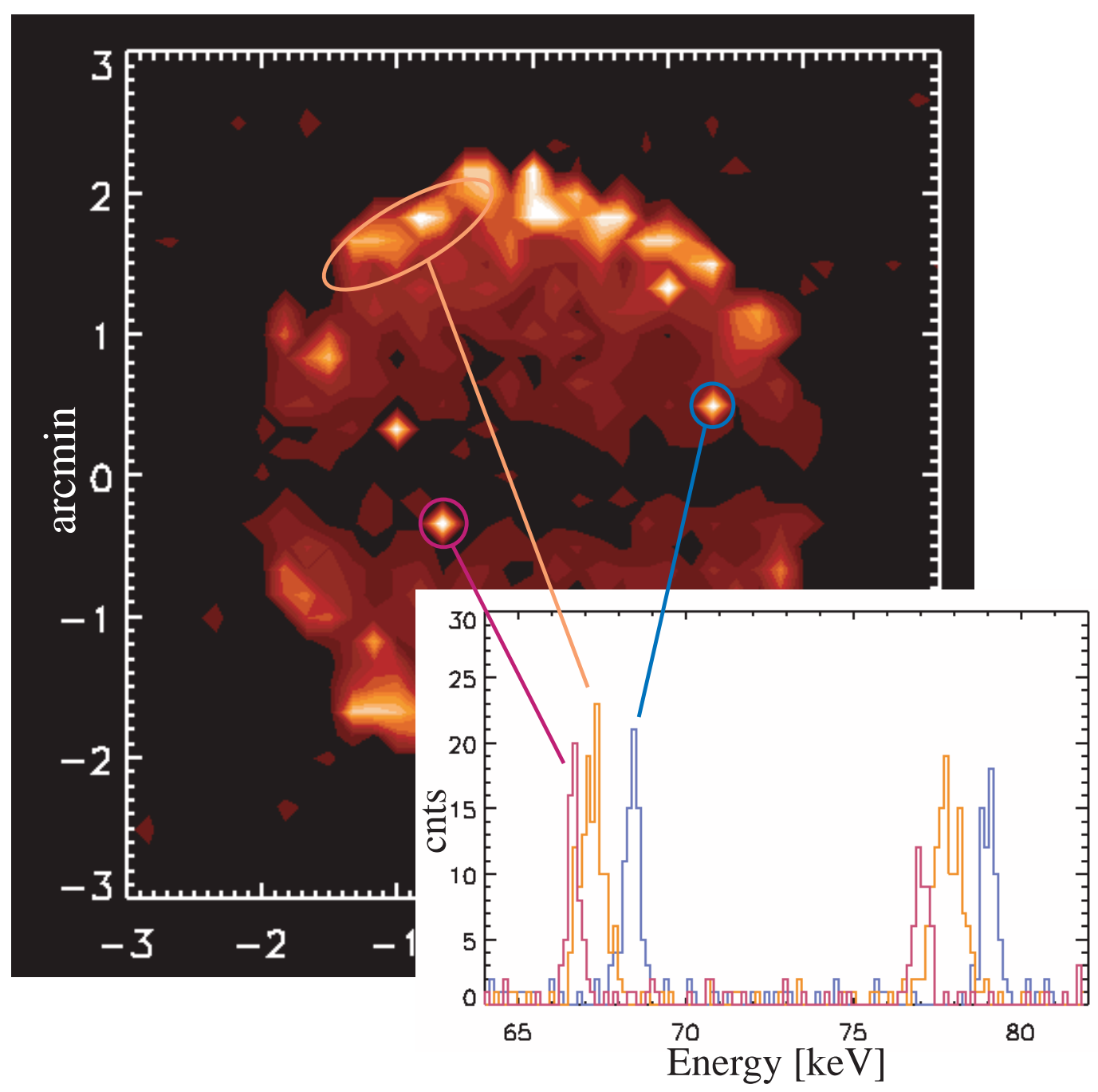

Figure 1. Simulation of a $10^{6} \mathrm{~s}$ exposure of the Cas A SNR in the 68 and $78 \mathrm{keV}$ lines of ${ }^{44}$ Ti. The simulation assumes a (clearly resolved) clumpy, axisymmetric explosion. Shown are the spectra of individual clumps, assuming ejection velocities with a $1000 \mathrm{~km} / \mathrm{s}$ spread. Bright knots can be clearly resolved on $10^{\prime \prime}$ scales, and relative ejecta velocities measured to better than $500 \mathrm{~km} / \mathrm{s}$. 
Comptel detected ${ }^{44} \mathrm{Ti} 1157 \mathrm{keV}$ line in the Cas A remnant, ${ }^{6}$ finding a surprisingly high flux given that the explosion, as described in historical records, was not that bright. Theory now suggests this may be due to asymmetry in the explosion. At $\mathrm{MeV}$ wavelengths, even future, advanced missions will not resolve the remnant, or map the velocity distribution - the information required to really constrain theoretical explosion models. Using the lower energy lines, however, HSI will be able to make detailed maps of Cas A and other young remnants. This is one of the few ways currently conceivable to observationally constrain models of stellar collapse. SN1987A is another target easily detectable by HSI, even for fluxes a factor ten below theoretically predicted values. This observation provides the exiting possibility of studying the line in an event where the progenitor is known, and where exquisite HST and other data exists.

\subsection{Obscured AGN and the Accretion Luminosity of the Universe}

By extending coverage to $10 \mathrm{keV}$, Chandra and XMM are beginning to uncover the population of dust-enshrouded, obscured AGN. These objects, difficult to detect in optical surveys due to the high internal extinction, appear to account for up to $80 \%$ of the accretion energy output in the universe. These missions are, however, only seeing the tip of the iceberg. Spectral coverage up to $100 \mathrm{keV}$ is required to both detect Compton thick sources, and to measure the continuum above the absorption cutoff $(\sim 10-15 \mathrm{keV})$ necessary to understand the geometry and internal energy output of the central black hole. HSI's sensitivity, arcsecond localizations, and spectral coverage (extending to $5 \mathrm{keV}$, so that absorbing column depths can be measured) will enable a large sample of such objects from the Swift, and EXIST catalogs to be localized well enough to find counterparts in order to identify the AGN, measure distances, and therefore understand the frequency, distribution and luminosity of obscured sources in the universe.

More than just an issue of taxonomy, determining the total accretion luminosity of massive black holes may have major implications for understanding the formation and evolution of galaxies. If, as it now appears, this is comparable to that associated with stellar emission, this factor must be included in current models of galaxy formation.

Although the Con-X Hard X-ray telescope, which extends coverage to $40 \mathrm{keV}$, will likely observe a few unidentified sources from the Swift catalog, it almost certainly will not observe the large sample required to address this question.

\subsection{Compact Objects in the Local Group and Other Objectives}

$H S I$ has the sensitivity and spectral resolution to perform unique investigations of a variety of compact stellar remnants within the Local Group of galaxies. Objects with hard spectra, well-suited to HSI are (1) high $\left(\gtrsim 10^{12}\right.$ Gauss) magnetic field X-ray pulsars, to study a variety of compact stellar remnants, (2) low-luminosity accretion onto weakly-magnetic neutron stars in low-mass binaries, and (3) transient emission from black hole candidates with both high and low-mass companions. These sources both inform us about the endpoints of stellar evolution in binaries, and sample stellar populations as a function of the Hubble type of the host galaxy.

With HSI spectral resolution, it can perform detailed studies of cyclotron scattering resonance features in highly magnetized pulsars, providing a direct measurement of the surface magnetic field strength, the spatial distribution of the field, the geometry of the accretion flow, and the plasma optical depth and temperature, which can be used to set limits on the luminosity and the distance of the source as well as the accretion rate. HSI's spatial resolution and broad bandpass will enable it to identify and study accreting sources out to M31.

The objectives listed above will constitute a major part of HSI's observing program, scheduled as transient followup and major surveys. In addition, some fraction of HSI's observing time will be devoted to guest investigators. An example of individual, pointed investigations include mapping the magnetic field strength in galaxy clusters through detection of inverse Compton scattered CMB photons, mapping diffuse hard emission in the Galactic center, constraining past accretion from the central black hole, mapping pulsar winds in the Crab Nebula, and resolving non-thermal continuum emission in young supernova remnants, probing shock acceleration and the origin of cosmic rays, and measuring the gamma-ray burst afterglow emission, including iron lines, in the X-ray band, constraining models for the relativistic shocks, and providing a probe of the density of the surrounding medium.

\section{HSI MISSION REQUIREMENTS}

The primary scientific goals driving the $H S I$ design are the measurements of prompt line emission from Type 1a SNe, high-spatial resolution mapping of ${ }^{44} \mathrm{Ti}$ emission in young remnants, and sensitive, high-positional accuracy studies of AGN. Table 1 lists the corresponding instrument requirements. Achieving these goals requires an array of focusing 

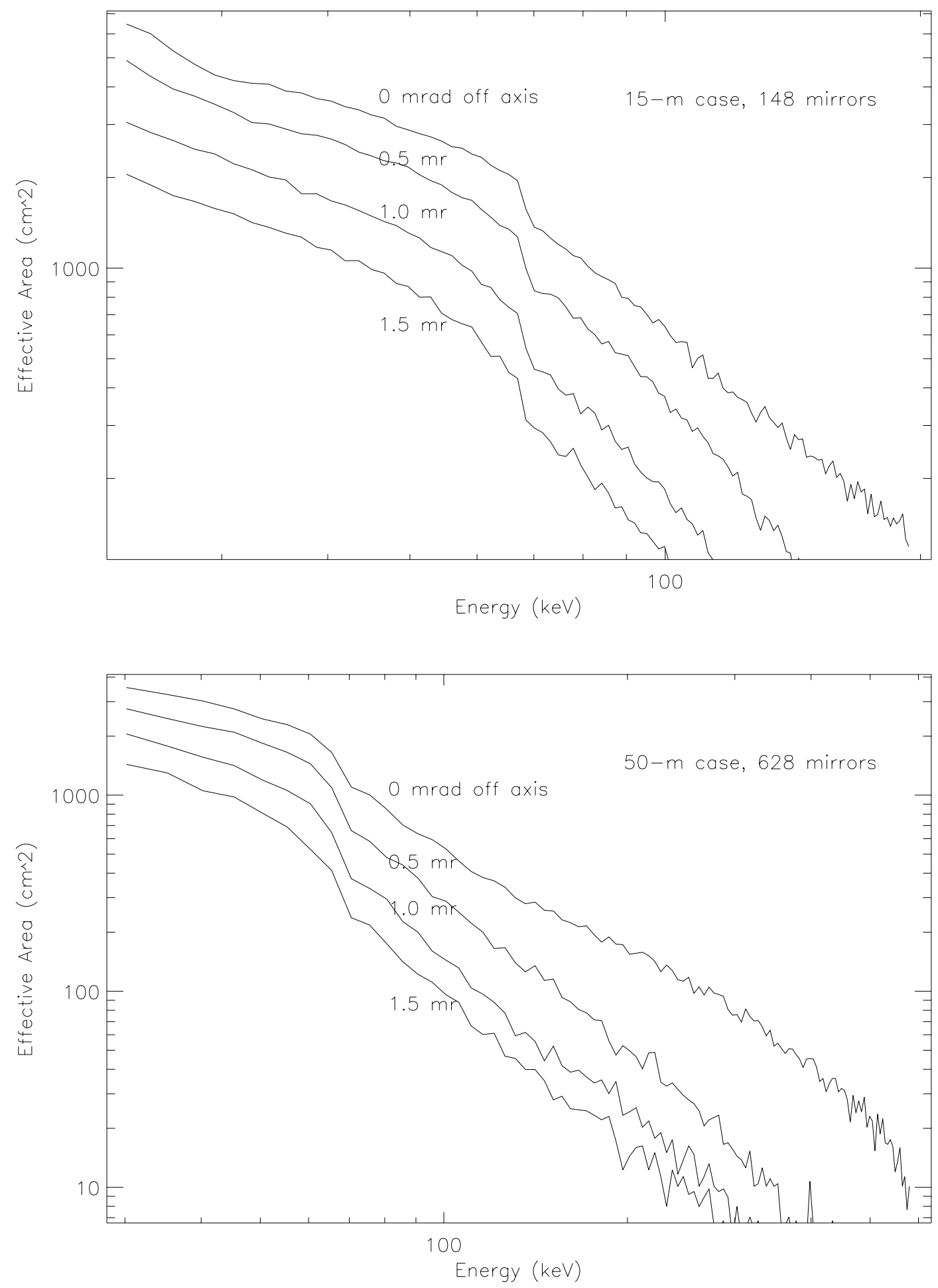

Figure 2. The calculated effective area for a focal length of 15 and $50 \mathrm{~m}$. The area is calculated for on-axis, 0.5 mrad off-axis, $1.0 \mathrm{mrad}$ off-axis and $1.5 \mathrm{mrad}$ off axis. The figure demonstrates the advantage of long focal length, and shows that for $50 \mathrm{~m}$, the effective area can extend to $600 \mathrm{keV}$. See Christensen et al. $(2000)^{7}$ for details of the optics design. 


\begin{tabular}{|l||l|}
\hline Energy range & $5-200 \mathrm{keV}$ (required) \\
& $5-600 \mathrm{keV}$ (goal) \\
\hline Angular resolution & $20^{\prime \prime}$ \\
\hline Source localization & $1^{\prime \prime}$ \\
\hline Collecting area & $2000 \mathrm{~cm}^{2}(\mathrm{E}<50 \mathrm{keV})$ \\
& $1000 \mathrm{~cm}^{2}(68 \mathrm{keV})$ \\
& $1500 \mathrm{~cm}^{2}(158-170 \mathrm{keV})$ \\
\hline Concentration factor & $>10^{4}$ \\
\hline Sensitivity & $<10^{-7} \mathrm{~cm}^{-2} \mathrm{~s}^{-1}$ \\
& $<0.1 \mu \mathrm{crab}($ continuum $5-100 \mathrm{keV})$ \\
& $<0.5 \mu \mathrm{crab}($ coninuum $100-200 \mathrm{keV})$ \\
\hline Energy resolution & $0.3 \mathrm{keV} \mathrm{@} 68 \mathrm{keV}$ \\
& $1 \mathrm{keV} @ 158 \mathrm{keV}$ \\
\hline FOV & 10 arcmin $(68 \mathrm{keV})$ \\
\hline Timing accuracy & $1 \mu \mathrm{s}$ \\
\hline Response time to transients & $<0.5$ day \\
\hline
\end{tabular}

Table 1. HSI instrument requirements

\begin{tabular}{|lc||l|}
\hline Graded-multilayer coated conical approximation to & \\
Wolter-I telescopes & focal length \\
& \# telescope modules \\
& graze angles & $30-50 \mathrm{~m}$ \\
& $6-12$ \\
& & \\
& & \\
& & \\
Solid state Ge pixel detectors & pixel size & $0.7-1.4 \mathrm{~mm}$ \\
with low-noise readout & diameter & $10-15 \mathrm{~cm}$ \\
& & AEC Able SRTM mast \\
\hline Extendible optical bench & LEO or L2 \\
\hline Orbit & 5 years \\
\hline Mission lifetime & Delta 7900 series \\
\hline Launch vehicle & \\
\hline
\end{tabular}

Table 2. HSI instrument parameters

telescopes using multilayer-coated optics and solid state germanium pixel detectors with a low-noise readout. The telescopes require a long focal length $(25-50 \mathrm{~m}$ ) in order to achieve the required collecting area up to $\sim 500 \mathrm{keV}$ (see Figure 2). With a $50 \mathrm{~m}$ focal length, which is achievable for some orbit choices, the telescope sensitivity will extend to $600 \mathrm{keV}$, thereby including the annihilation line region. Table 2 summarizes the instrument parameters.

The primary mission life will be 5 years, however the focal plane will be electrically cooled, with no expendables other than for reorientation, so that a 10 year mission is not precluded. Much of the HSI mission will be devoted to large surveys (of AGN and SNR), and follow-up of supernovae discovered on the ground. Thus, scheduling and operations will be relatively simple. The final 1.5 years of the mission would be devoted to guest investigations.

\section{MISSION AND TECHNOLOGY DEVELOPMENT}

The HSI instrument is based on an array of Wolter I (or conical approximation) grazing incidence optics and solid state germanium pixel detectors. The optics are coated with depth-graded multilayers to extend the experiment bandpass to high energy. The optics and multilayer development builds on systems developed for the Constellation$X \mathrm{HXT}^{8}$ and $H E F T^{9}$ balloon experiments. The high-resolution, high-energy extension of this technology required by $H S I$ is described in other papers (see Windt et al. (2000), ${ }^{10}$ Windt et al. (2002) and Hailey et al. (2002), these 


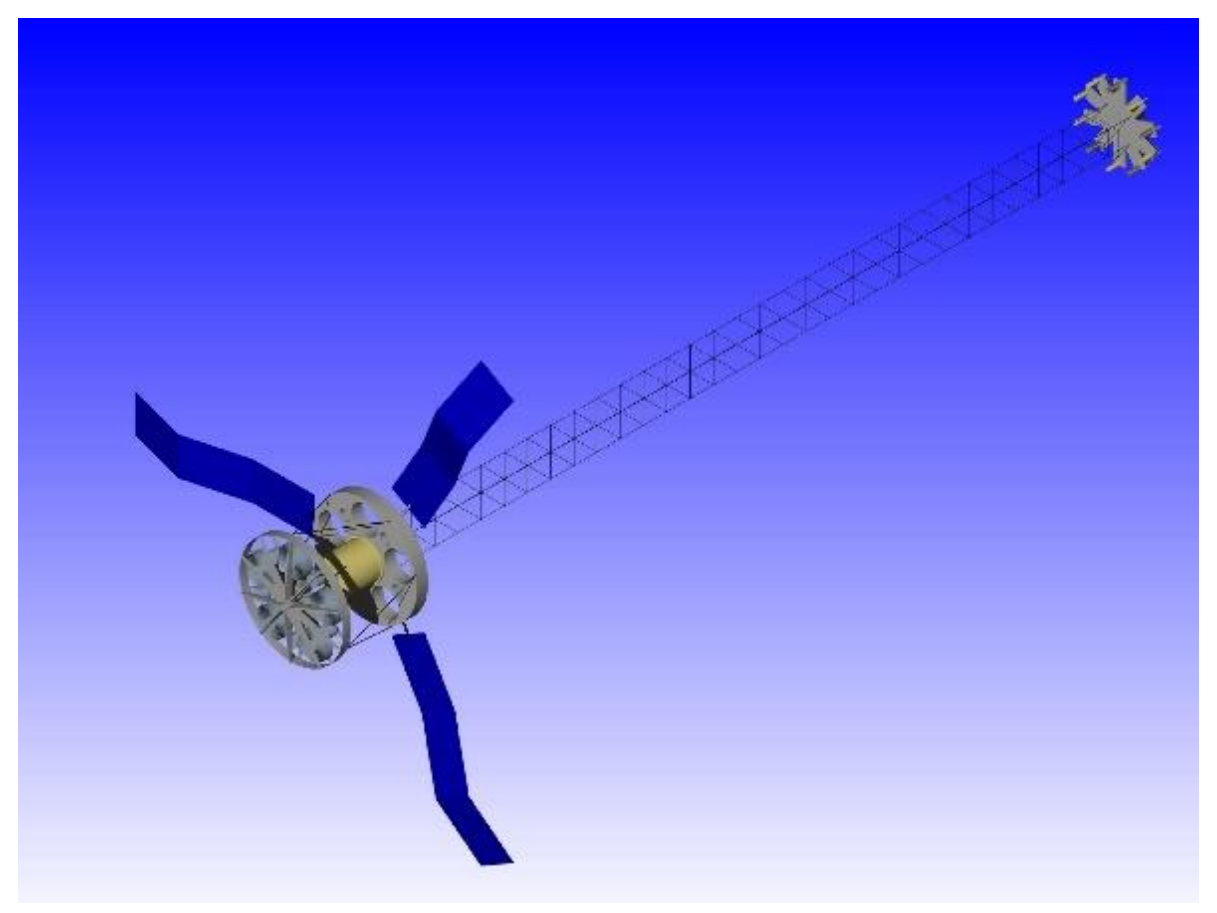

Figure 3. Schematic of the HSI spacecraft. An extendable optical bench maintains a $50 \mathrm{~m}$ focal length. The spacecraft surrounds the optics assembly, while the lower-mass detector assembly is deployed.

proceedings). Christensen et al. $(2000)^{7}$ and Chen et al. (2002), these proceedings, have described appropriate multilayer designs for high-energy application. The detectors require cryogenic low-noise, low-power ASICs to be developed, as well as improvements in contact technologies. Table 2 lists the instrument parameters.

The final configuration (focal length, optics and detector dimensions) is still under study, and will largely depend on spacecraft considerations.

\section{CONCLUSION}

$H S I$ will focus energetic X-rays and low-energy gamma-rays will enable it to observe the high-energy cosmos with an unprecedented combination of sensitivity, spectral resolution, and angular resolving power. This will allow significant scientific advances in a number of diverse areas of astrophysics. In particular, HSI's capabilities will allow sensitive, high spatial and spectral resolution observations of key nuclear transitions in Type 1a and core-collapse SNe for the first time. The mission is of moderate size, and the technologies are relatively modest extrapolations of existing systems.

\section{REFERENCES}

1. J. Grindlay, L. Bildsten, R. Blandford, D. Chakrabarty, M. Elvis, A. Fabian, F. Fiore, G. Fishman, N. Gehrels, C. Hailey, F. Harrison, D. Hartmann, C. Kouveliotou, T. Prince, B. Ramsey, R. Rothschild, G. Skinner, and S. Woosley, "EXIST: The Ultimate Spatial/Temporal Hard X-ray Survey," in Gamma 2001: GammaRay Astrophysics, held 4-6 April, 2001 in Baltimore, MD. Edited by Steven Ritz, Neil Gehrels, and Chris R. Shrader. Also AIP Conference Proceedings, Vol. 587. Melville, NY: American Institute of Physics, 2001., p.899, pp. 899-+, 2001.

2. A. Tyson and R. Angel, "The Large-aperture Synoptic Survey Telescope.," in ASP Conf. Ser. 232: The New Era of Wide Field Astronomy, pp. 347-+, 2001.

3. S. E. Woosley and T. A. Weaver 24, p. 205, 1986.

4. K. W. Chan and R. E. Lingenfelter ApJ 368, p. 515, 1991.

5. A. Burrows and L. S. The ApJ 360, p. 626, 1990. 
6. A. F. Iyudin et al. Nature 396, p. 142, 1998.

7. F. E. Christensen, J. M. Chakan, F. A. Harrison, S. E. Boggs, P. H. Mao, T. A. Prince, W. W. Craig, C. J. Hailey, and D. L. Windt, "Grazing incidence optics designs for future gamma-ray missions," in Proc. SPIE Vol. 4012, p. 278-283, X-Ray Optics, Instruments, and Missions III, Joachim E. Truemper; Bernd Aschenbach; Eds., vol. 4012, pp. 278-283, July 2000.

8. F. A. Harrison, W. R. Cook, F. E. Christensen, O. Citterio, W. W. Craig, N. A. Gehrels, P. Gorenstein, J. E. Grindlay, C. J. Hailey, R. A. Kroeger, H. Kuneida, G. Pareschi, A. M. Parsons, R. Petre, and S. E. Romaine, "Technology development for the Constellation-X hard-x-ray telescope," in Proc. SPIE Vol. 3765, p. 104-111, EUV, X-Ray, and Gamma-Ray Instrumentation for Astronomy X, Oswald H. Siegmund; Kathryn A. Flanagan; Eds., vol. 3765, pp. 104-111, Oct. 1999.

9. F. A. Harrison, S. E. Boggs, A. E. Bolotnikov, F. E. Christensen, W. R. Cook, W. W. Craig, C. J. Hailey, M. A. Jimenez-Garate, P. H. Mao, S. M. Schindler, and D. L. Windt, "Development of the High-Energy Focusing Telescope (HEFT) balloon experiment," in Proc. SPIE Vol. 4012, p. 693-699, X-Ray Optics, Instruments, and Missions III, Joachim E. Truemper; Bernd Aschenbach; Eds., vol. 4012, pp. 693-699, July 2000.

10. D. L. Windt, F. E. Christensen, W. W. Craig, C. J. Hailey, F. A. Harrison, M. A. Jimenez-Garate, R. Kalyanaraman, and P. H. Mao, "X-ray multilayer coatings for use at energies above $100 \mathrm{keV}$," in Proc. SPIE Vol. 4012, p. 442-447, X-Ray Optics, Instruments, and Missions III, Joachim E. Truemper; Bernd Aschenbach; Eds., vol. 4012, pp. 442-447, July 2000. 